\title{
Exposure to Nitrogen Oxide in the First Trimester and Risk of Cardiovascular-Related Malformations: A Dose-Response Meta-Analysis of Observational Studies
}

\author{
Tie-Ning Zhang, ${ }^{1}$ Da Li, ${ }^{2}$ Qi-Jun Wu, ${ }^{3}$ Jing Xia, ${ }^{1}$ Ri Wen, ${ }^{1}$ Xing-Chen Chen, \\ Ni Yang, ${ }^{1}$ Yan-Ling Chen, ${ }^{5}$ Yan-Hong Huang, ${ }^{6}$ and Chun-Feng Liu $\mathbb{D}^{1}$ \\ ${ }^{1}$ Department of Pediatrics, Shengjing Hospital of China Medical University, Shenyang, China \\ ${ }^{2}$ Department of Obstetrics and Gynecology, Shengjing Hospital of China Medical University, Shenyang, China \\ ${ }^{3}$ Department of Clinical Epidemiology, Shengjing Hospital of China Medical University, Shenyang, China \\ ${ }^{4}$ Department of Neurosurgery, Shengjing Hospital of China Medical University, Shenyang, China \\ ${ }^{5}$ Liaoning Women and Children's Health Hospital, Shenyang, China \\ ${ }^{6}$ Department of Science and Education, Shenyang Women and Children Health Care Centre, Shenyang, China
}

Correspondence should be addressed to Chun-Feng Liu; zhliu258@hotmail.com

Received 14 September 2017; Revised 23 February 2018; Accepted 5 March 2018; Published 10 April 2018

Academic Editor: Hai-Feng Pan

Copyright (c) 2018 Tie-Ning Zhang et al. This is an open access article distributed under the Creative Commons Attribution License, which permits unrestricted use, distribution, and reproduction in any medium, provided the original work is properly cited.

\begin{abstract}
Nitrogen oxide $\left(\mathrm{NO}_{x}\right)$ is produced during combustion at high temperature, which is a major constituent of air pollutants. Recent studies suggested inconsistent results on the association between $\mathrm{NO}_{x}$ exposure and cardiovascular-related malformations. We aimed to assess aforementioned association in pregnant women in the first trimester and cardiovascular-related malformations of infants. A systematic literature review identified studies for observational studies about $\mathrm{NO}_{x}$ exposure and cardiovascular-related malformation in PubMed. Random-effect models were used to estimate summary odds ratio (SOR) and 95\% confidence intervals (CIs) for aforementioned association. Finally, nine studies met the inclusion criteria. Overall, the SOR of cardiovascular-related malformation per $10 \mathrm{ppb}$ increment in $\mathrm{NO}_{x}$ and $\mathrm{NO}_{2}$ concentration was 1.01 (95\% CI: 0.98-1.04; $\left.I^{2}=38.6 \%, P=0.09\right)$ and 0.99 (95\% CI: $\left.0.95-1.04 ; I^{2}=37.8 \%, P=0.13\right)$, respectively. Stratifying by study design, geographic locations, and confounded adjustments, the majority of strata showed negative results, which were consistent with the main findings. However, we found that exposure to $\mathrm{NO}_{x}$ and $\mathrm{NO}_{2}$ in the first trimester increased the risk of coarctation of the aorta (COA) malformation by $13 \%$ and $19 \%$, respectively. Our study provided limited evidence regarding the association between $\mathrm{NO}_{x}$ exposure in the first trimester and cardiovascular-related malformations in infants.
\end{abstract}

\section{Introduction}

Air pollution has become a major problem in many countries. Increasing evidence showed that air pollution could not only lead to global warming, but also have adverse effects on the fetus and newborn from maternal exposure $[1,2]$, such as preterm birth, low birth weight, and intrauterine growth retardation [3-6]. Of note, nitrogen oxide $\left(\mathrm{NO}_{x}\right)$, a major constituent of air pollutants, mainly refers to nitric oxide $(\mathrm{NO})$ and nitrogen dioxide $\left(\mathrm{NO}_{2}\right)$, which are produced during combustion, especially at high temperature. These two substances are important trace species in earth's atmosphere.
Congenital malformations are an important cause of infant mortality and a leading cause of disability worldwide [7]. Notably, cardiovascular-related malformation, which constitutes most common group of birth defects [8], has become the main cause of death in infants with congenital anomalies and is associated with a considerable burden on public [912]. For example, the previous study reported that congenital heart defects occur in 4-8 of 1000 live births [13], the most common being ventricular septal defects (VSD) $(27.5 / 10,000$ live births) and atrial septal defects (ASD) (10.6/10,000 live births) [14]. Although the etiology of cardiovascular-related malformation is still unclear, several studies suggested that it 
might be related to both genetic and environmental factors $[10,15]$. Additionally, recent studies have proposed that air pollution including nitrogen oxide could play an important role in causing cardiac defect $[2,16]$, because of its ability to act directly as prooxidants of lipid and proteins or as free radical generators promoting oxidative stress and the induction of inflammatory responses [17]. For example, a meta-analysis suggested that $\mathrm{NO}_{2}$ exposure was related to increases in risk of coarctation of the aorta (COA) and tetralogy of Fallot (TOF) [18], while the other one suggested that $\mathrm{NO}_{2}$ exposure was only associated with COA [19]. However, several limitations were observed in the previous meta-analyses: (1) the exposure period of $\mathrm{NO}_{x}$ of pregnant women was not consistent; for example, the meta-analysis carried out by Vrijheid et al. [18] included different exposure periods of $\mathrm{NO}_{x}$ (e.g., first trimester, second trimester, third trimester, and three-month preconception); (2) the results of subgroup analyses stratified by adjustment for potential confounders were limited; (3) although atrial septal defect (ASD) and ventricular septal defect (VSD) are the most common cardiovascular-related malformations [14], the previous meta-analyses did not perform throughout analyses regarding these two malformations and the evidence of the associations between $\mathrm{NO}_{x}$ and ASD or VSD was relatively finite.

In order to better understand the relationship between nitrogen oxide exposure in pregnant women in the first trimester and cardiovascular-related malformations of infants, we performed a systematic review and meta-analysis using data from epidemiologic studies.

\section{Methods}

2.1. Literature Search. This meta-analysis was planned, performed, and reported in adherence to the Meta-analysis of Observational Studies in Epidemiology (MOOSE) guideline [20]. We conducted computerized literature searches in the PubMed and reviewed the data from the database index through April 15, 2017. The following search key words and Medical Subject Heading (MeSH) terms were used: (air pollution OR nitrogen oxide OR nitrogen dioxide OR nitric oxide OR $\mathrm{NO}_{x} \mathrm{OR} \mathrm{NO}_{2}$ OR NO) AND (birth defect OR congenital anomalies OR cardiovascular-related malformation OR cardiac anomalies OR cardiac malformation OR congenital heart disease OR defect OR congenital abnormalities OR birth outcome OR obstetrical outcome). Additionally, the references cited in the retrieved articles were scrutinized by manual research.

2.2. Study Selection. The published studies were considered to be included if they met the following criteria: if they (i) used an epidemiologic study design (e.g., case-control, casecohort, and cohort study); (ii) defined the exposure period of nitrogen oxide as the first trimester or in the range of first trimester during pregnancy; (iii) reported cardiovascularrelated malformation as the outcomes of interest; (iv) reported the usable risk estimates (e.g., odd ratio, risk ratio, or relative risk with $95 \%$ confidence intervals (CIs) or necessary data for calculation) of the association between nitrogen oxide exposure and cardiovascular-related malformation.
The published studies were excluded by the following exclusion criteria: if they (i) were nonepidemiologic studies (e.g., case-control, case-cohort, and cohort study), review articles, systematic review and meta-analyses, commentaries, editorials, or meeting abstracts; (ii) reported the risk estimates that could not be summarized (such as reporting the risk estimates without 95\% CIs) or used for further unit conversion (such as reporting the risk estimates of $\mathrm{NO}_{x}$ with the unit of "microgram per cubic meter $\left(\mathrm{ug} / \mathrm{m}^{3}\right)$ "); (iii) reported the exposure period during the period of preconception or other trimesters; (iv) were not human studies or published in English.

When duplicate articles from the same study were identified, we selected the most recent report that contained the largest number of the cases or cohort that matched our interest. All abovementioned study selection and exclusion procedures were carried out by two independent researchers (TieNing Zhang and Qi-Jun Wu).

2.3. Data Extraction. Data was independently extracted according to a standardized format by two researchers (TieNing Zhang and Jing Xia) for each eligible study. Disagreements were discussed and solved by a third researcher (Qi-Jun Wu). The following important study characteristics were abstracted from each included study: the first author, year of publication, geographic location, study period, study design, number of cases, gestational period, kind of exposure, outcome with their risk estimates, and 95\% CIs. We also extracted the factors matched between cases and controls and potential confounders of each study. If there were multiple estimates of the association, we extracted the estimate that was adjusted for the largest number of potential confounders. If both single- and multiple-pollutant models were presented in a study, we selected single-pollutant model in our metaanalysis.

2.4. Quality Evaluation. Two independent researchers (TieNing Zhang and Jing Xia) conducted the quality assessment of included studies according to Newcastle-Ottawa Scale (NOS) for cohort and case-control studies. All 8 items in the scale were applicable to our study question. The items can be divided into 3 domains (e.g., selection, comparability, and exposure/outcome). We used these NOS parameters to evaluate the studies instead of scoring them and categorizing them into high or low quality on the basis of the scores [2123].

2.5. Statistical Analysis. As the absolute risk of cardiovascular-related malformation is low, we reported all results in terms of OR for simplicity. Considering there was limited evidence regarding $\mathrm{NO}$ and cardiovascular-related malformation, we just examined the relationship between $\mathrm{NO}_{x}$ or $\mathrm{NO}_{2}$ and cardiovascular-related malformation. Firstly, we converted the unit of $\mathrm{ug} / \mathrm{m}^{3}$ into part per billion (ppb) in order to allow comparison of effects among different studies. Subsequently, we summarized and converted the studyspecific ORs for each $10 \mathrm{ppb}$ increment in $\mathrm{NO}_{x}$ or $\mathrm{NO}_{2}$ concentration. For conducting dose-response meta-analysis, the study-specific trend from the correlated log ORs across the 
categories of $\mathrm{NO}_{x}$ or $\mathrm{NO}_{2}$ concentrations was computed by using the generalized least-squares trend estimation method developed by Greenland and Longnecker [24] and Orsini et al. [25]. If the study results were presented as a quantitative exposure category, for conducting dose-response metaanalysis, we required information on (i) the distribution of cases and noncases and risk estimates with variance estimates for at least three quantitative exposure categories, (ii) median or mean level of these exposures in each category (if reported based on range, the mean level was calculated by averaging the lower and upper boundary; if the lowest category was open-ended, the lowest boundary was considered zero; if the highest category was open-ended, the open-ended interval length was assumed to be the same as the adjacent interval).

To examine the associations between the exposure (e.g., $\mathrm{NO}_{x}$ and $\mathrm{NO}_{2}$ ) and the outcomes of our interest, the summary odds ratio (SOR) with 95\% CIs was estimated by summarizing the risk estimates of each study using the random-effect models [26]. For the study [27-31] that separately reported several kinds of outcomes but did not combine them (e.g., the risk estimates of ASD, conotruncal defects, and transposition of the great arteries), we recalculated total risk estimates as that of cardiovascular-related malformation using fixed effect model. Because several studies [7, 27-34] reported the outcomes of $\mathrm{ASD}, \mathrm{VSD}, \mathrm{TOF}$, and COA, we extracted this data in order to calculate the SORs of these events.

Heterogeneity among studies was assessed with $I^{2}$ statistics, and an $I^{2}$ value greater than $50 \%$ was considered to indicate substantial heterogeneity. To investigate the possible sources of heterogeneity of main results, we performed stratified analyses by the following study features: study design (cohort study versus case-control study), geographic location (US versus others), and potential confounders considered or adjusted for in the analyses (maternal age, socioeconomic status, conception season, year of birth, infant sex, gestational age, and educational level). Heterogeneity between subgroups was evaluated by meta-regression analysis. We also performed sensitivity analyses by excluding one study at a time to explore whether results were strongly influenced by a specific study.

Finally, publication bias was evaluated through Egger et al.s test [35] and Begg and Mazumdar's test [36]. We assumed that there was a significant statistical publication bias if $P$ value is less than 0.05 for Egger's or Begg's test. All statistical analyses were performed with Stata 12.1 (StataCorp).

\section{Results}

3.1. Literature Search. Our literature search process is illustrated in Figure 1. Briefly, our initial search yielded 5,595 studies from PubMed, of which we screened the titles and abstracts. After a further review and evaluation, 5,564 studies were excluded for the general criteria. The remaining 31 studies were identified through detailed assessment. In the end, nine studies [7, 27-34] were eligible for inclusion criteria and considered into our final dose-response meta-analysis.

3.2. Study Characteristics. The characteristics of the eight studies are summarized in Tables S1 and S2. Briefly, these studies were published between 2009 [28, 29] and 2015 [27]. Of all these included studies, three studies each were conducted in America [29-31], two studies were conducted in Israel [7, 33], and one study was conducted in China [27], Spain [34], Northeast England [32], and Australia [28], respectively. Besides, we included three cohort studies [7, 29, $33]$ and six case-control studies [27, 28, 30-32, 34]. Among case-control studies, five studies $[27,28,31,32,34]$ were population-based and another [30] was hospital-based. The number of cardiovascular-related malformations varied from 572 [28] to 4,639 [29], and the total number of cases was 19,079 .

3.3. Quality of Included Studies. Tables S3a and S3b present the study-specific quality according to Newcastle-Ottawa quality scale. As for cohort studies, in the "follow-up long enough for outcomes to occur" and "adequacy of follow-up of cohorts" categories, no studies were assigned scores because these three cohort studies were all retrospective. The maximum score is seven [7] and the minimum score is five [29]. As for case-control studies, in the "non-response rate" categories, three studies were not assigned score because they did not refer to non-response rate or provide available data for calculation. The maximum score is nine [27] and the minimum score is six [28].

3.4. $\mathrm{NO}_{x}$ and Cardiovascular-Related Malformation per $10 \mathrm{ppb}$ Increment. Nine studies [7, 27-34] were included in the analysis of the association between $\mathrm{NO}_{x}$ concentrations and cardiovascular-related malformation (Figure 2, Table 1). Overall, the SOR of cardiovascular-related malformation per $10 \mathrm{ppb}$ increment in $\mathrm{NO}_{x}$ concentration was 1.01 (95\% CI: $0.98-1.04)$, with low heterogeneity $\left(I^{2}=38.6 \%, P=0.09\right)$. There was no indication of a publication bias according to Begg's test $(P$-bias $=1.00)$ and Egger's test $(P$-bias $=0.65)$.

Furthermore, analyses of studies reported the cardiac malformation of interest that were ASD [7, 27, 28, 30-34], VSD [7, 27-34], TOF [27, 29-32, 34], and COA [29-32, 34] with corresponding OR scores of 1.01 (95\% CI: 0.96-1.06), 1.03 (95\% CI: 0.97-1.10), 1.04 (95\% CI: 0.98-1.11), and 1.13 (95\% CI: 1.05-1.22), respectively (Figure 3).

The results of stratified analyses by study characteristics are summarized in Table 1. When we performed subgroup analyses in terms of study design, geographic locations, and confounded adjustments, all strata showed negative results in the outcome of cardiovascular-related malformation and ASD, which were consistent with the main findings. As for the outcome of VSD stratified by study design, the point estimate for cohort study (SOR: 1.02; 95\% CI: 1.00-1.05) was slightly higher than that for case-control study (SOR: 1.01; $95 \%$ CI: 0.93-1.09), which suggested a mild positive association between $\mathrm{NO}_{x}$ exposure and VSD in cohort studies. Stratifying by adjustment for educational level, we observed significant results for cardiovascular-related malformation with low heterogeneity after summarizing three studies adjusted for this potential confounder. In addition, there was no evidence of significant heterogeneity between subgroups detected by meta-regression analyses. 


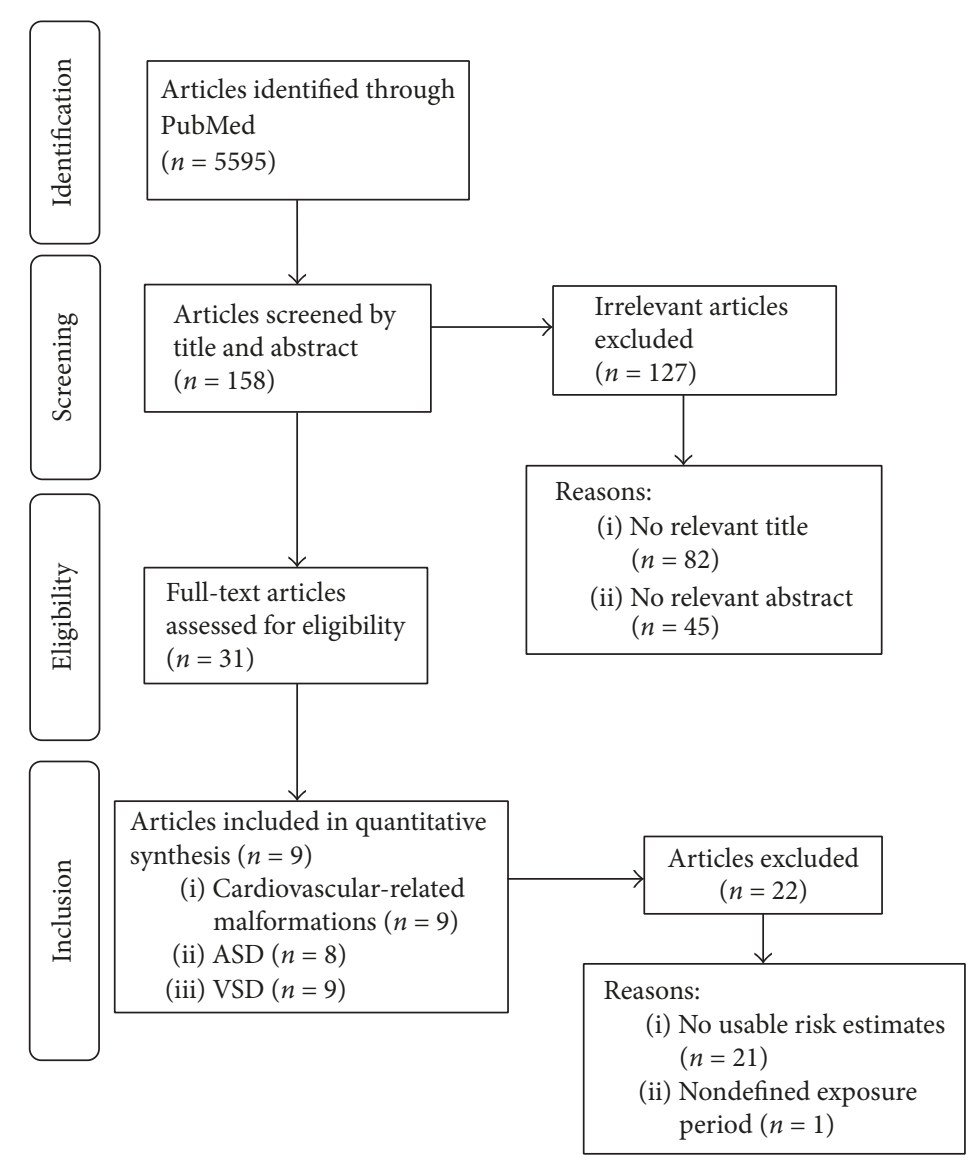

FIGURE 1: Flow chart of study selection.

In a sensitivity analysis, we evaluated the effect of removing a single study from the total in order to determine its effect on the summarized estimate for heterogeneity and to assess whether one study had a significant influence on the meta-analytic OR. The study-specific OR of cardiovascularrelated malformation ranged from a low value of 1.00 (95\% CI: $\left.0.90-1.02 ; I^{2}=0.0 \%, P=0.56\right)$ after omission of the study by Stingone et al. [31] to a high value of 1.01 (95\% CI: $\left.0.99-1.04 ; I^{2}=16.2 \%, P=0.30\right)$ after omission of the study by Padula et al. [30].

\section{5. $\mathrm{NO}_{2}$ and Cardiovascular-Related Malformation per $10 \mathrm{ppb}$} Increment. Eight studies [7, 27-32, 34] were included in the analysis of the association between $\mathrm{NO}_{2}$ concentration and cardiovascular-related malformation (Figure 4, Table 2). Overall, the SOR of cardiovascular-related malformation per $10 \mathrm{ppb}$ increment in $\mathrm{NO}_{2}$ concentration was 0.99 (95\% CI: $0.95-1.04)$, with low heterogeneity $\left(I^{2}=37.8 \%, P=0.13\right)$. There was no indication of publication bias with Egger's test $(P$-bias $=0.39)$ and Begg's test $(P$-bias $=0.20)$.

In addition, analyses of studies reported the cardiac malformations of interest that were ASD [7, 27-32, 34], VSD $[7,27-32,34]$, TOF $[27,29-32,34]$, and COA $[29-32,34]$ with corresponding OR scores of 0.99 (95\% CI: 0.93-1.06), 1.01 (95\% CI: 0.95-1.08), 1.03 (95\% CI: 0.95-1.11), and 1.19 (95\%
CI: 1.08-1.30), respectively (Figure 5). Of note, there was a significant increase risk of COA in infants born to mothers who are exposed to $\mathrm{NO}_{2}$ in the first trimester.

Stratifying by study design, geographic locations, and confounded adjustments, the majority of strata showed negative results in the outcome of cardiovascular-related malformation, ASD, and VSD, which were consistent with the main findings. There was no evidence of significant heterogeneity between subgroups detected by meta-regression analyses. In a sensitivity analysis omitting one study at a time and after we analyzed the SOR of the rest, the SORs of cardiovascularrelated malformation ranged from 0.98 (95\% CI: 0.94-1.02; $\left.I^{2}=0.0 \%, P=0.51\right)$ after excluding the study by Stingone et al. [31] to 1.00 (95\% CI: $\left.0.96-1.05 ; I^{2}=34.6 \%, P=0.16\right)$ after excluding the study by Dadvand et al. [32].

\section{Discussion}

Overall, the findings from this meta-analysis indicated there was no obvious increased risk between $\mathrm{NO}_{x} / \mathrm{NO}_{2}$ exposure and cardiovascular-related malformations. The same negative results were also observed in most subgroup analyses. However, we found that exposure to $\mathrm{NO} x$ and $\mathrm{NO}_{2}$ in the first trimester increased the risk for COA malformation by $13 \%$ (1.05-1.22) and 19\% (95\% CI: 1.08-1.30), respectively. 


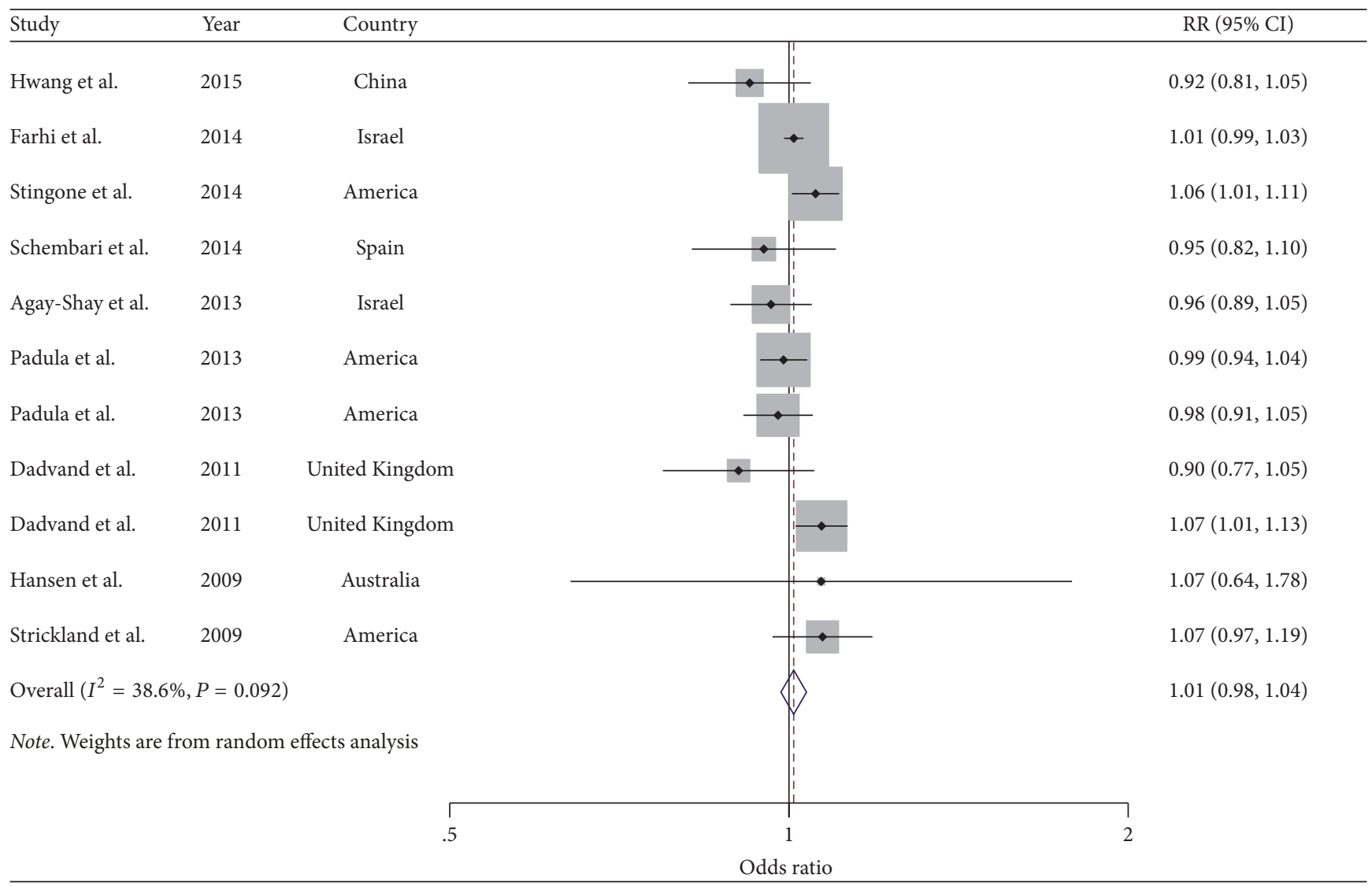

FIGURE 2: Forest plots of the relationship between $\mathrm{NO}_{x}$ exposure and risk of cardiovascular-related malformations. Squares indicate studyspecific risk estimates (size of the square reflects the study-specific statistical weight); horizontal lines indicate $95 \%$ CIs; diamond indicates the summary relative risk with its $95 \%$ CI.

The cause and mechanism of cardiovascular-related malformation still remain unclear. A study conducted by Wilhelm and Ritz [37] suggested that $\mathrm{NO}_{2}$ could oxidize tissue composition, increase lipid peroxidation in fetal and maternal vivo, and inhibit the protective action of the antioxidant defense system, which can affect fetal growth and development. Additionally, there was evidence that air pollution could also contribute to epigenetic change, including alteration of DNA methylation [38]. Besides, microRNA has been also studied with regard to the environmental changes and suggested that microRNA expression and regulation may be affected by environmental exposures [39]. These findings pointed out that epigenetic modifications during pregnancy could impair normal embryo development and cause cardiac defects. However, our meta-analysis found no significant increase in risk of most cardiovascular-related malformation with $\mathrm{NO}_{x}$ or $\mathrm{NO}_{2}$ exposure, which might result from exposure assessment of individual study. For example, exposure indices were usually calculated from pollutant measurements at the nearest monitoring station or as a distanceweighted average of measurements of all stations in the area. These methods apply a similar exposure to a relatively large geographic area and thereby measure predominantly community-wide variations in air pollution [18].
Considering traffic exhaust fumes are the main source of $\mathrm{NO}_{x}$ and $\mathrm{NO}_{2}$, this approach is not completely suitable for nitrogen oxide assessment, which may have a much finer spatial distribution. Power may be improved by using more personal measures of $\mathrm{NO}_{x}$ exposure and the ideal solution is to set monitors in a cohort of pregnant women, but this method would be very expensive [28]. A cheaper alternative is to measure the road network surrounding the pregnancy's home based on their geocoded address, as a proxy measure of pollution [28]. This raises an important issue and may guide further study design and exposure assessment.

It is possible that $\mathrm{NO}_{x}$ exposure might be associated with cardiovascular-related malformations, but this association is too small to detect because there were several known and unknown factors that might influence the results of individual study. Considering each included study was based on the data from registry or hospital program, the condition of personal exposure might be unpredictable and could be related to specific factors such as behavior pattern, living activity, working history, and indoor air pollution. Nondifferential errors were assumed between cases and controls, and therefore this exposure misclassification could influence effect estimate. For example, if mothers of included cases had more difficult pregnancies, this could limit their outdoor 


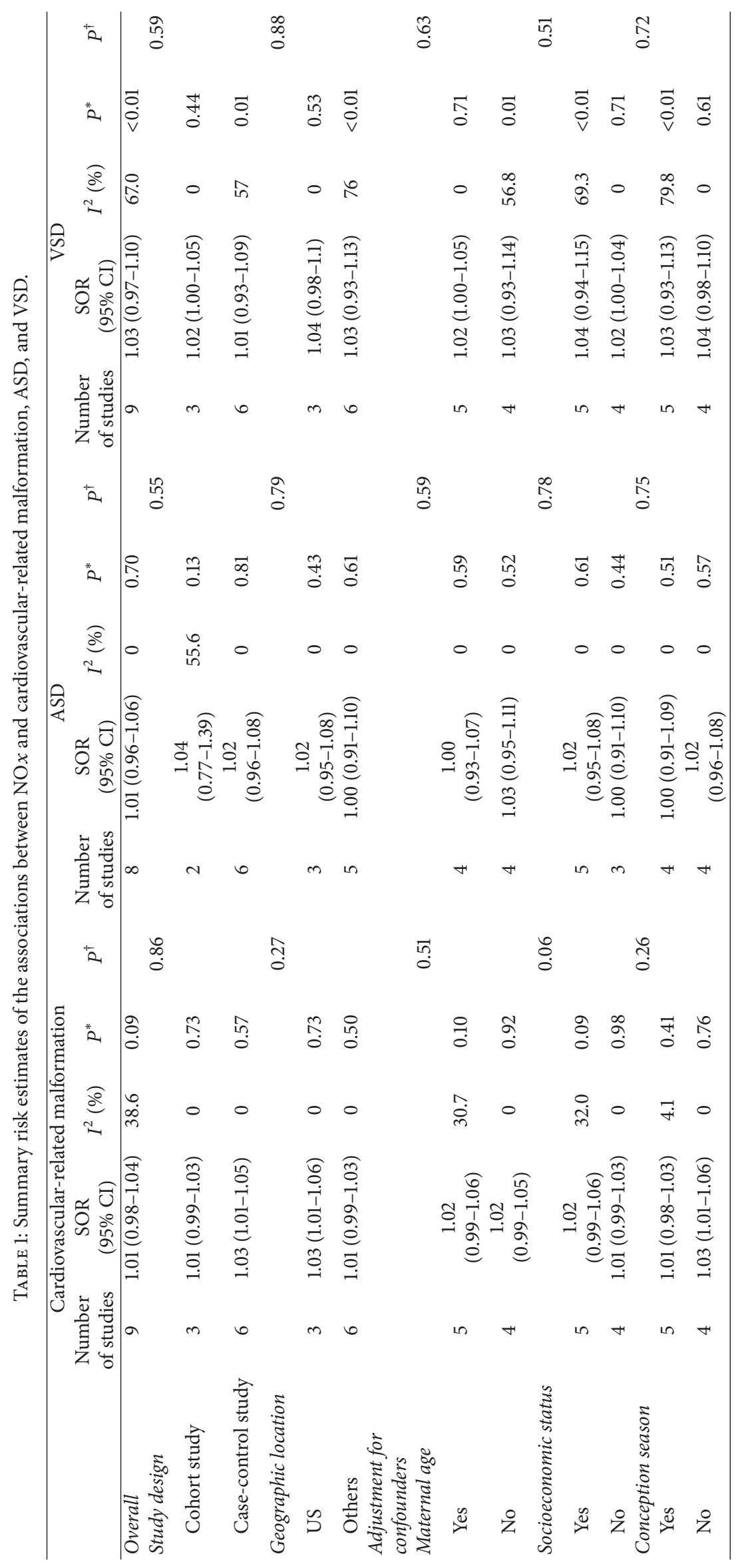




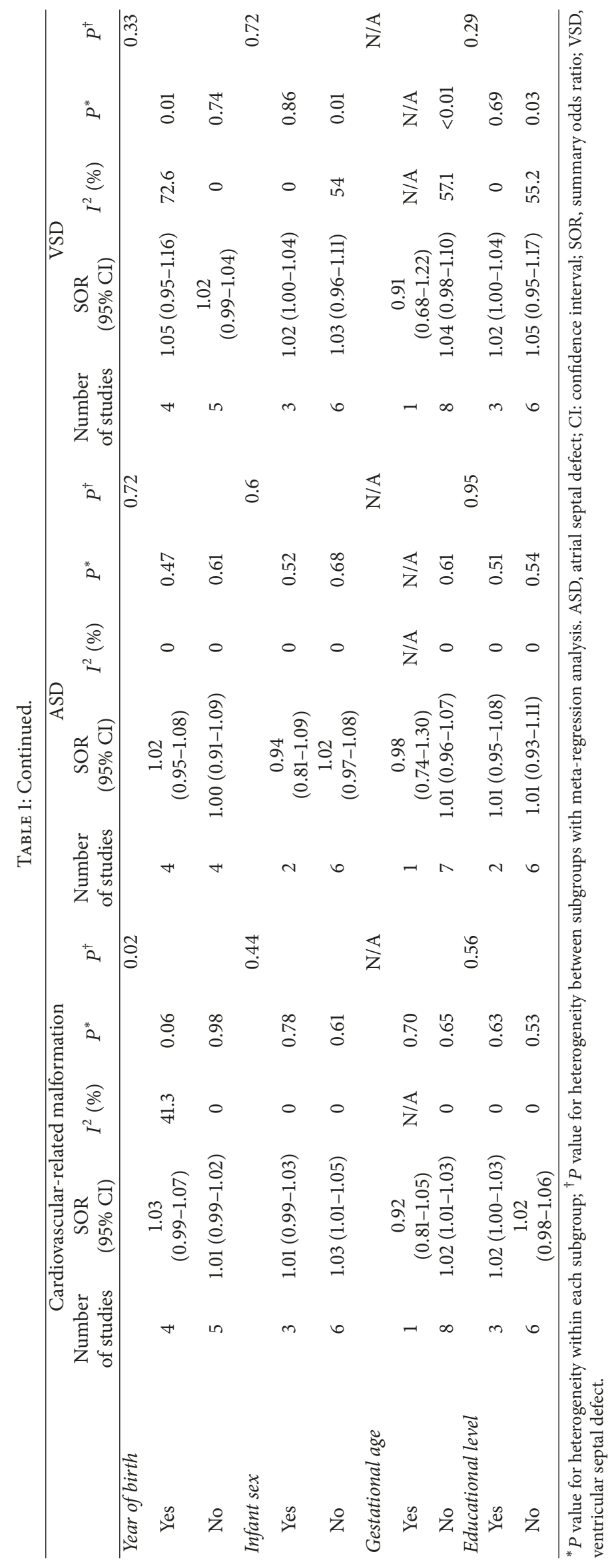




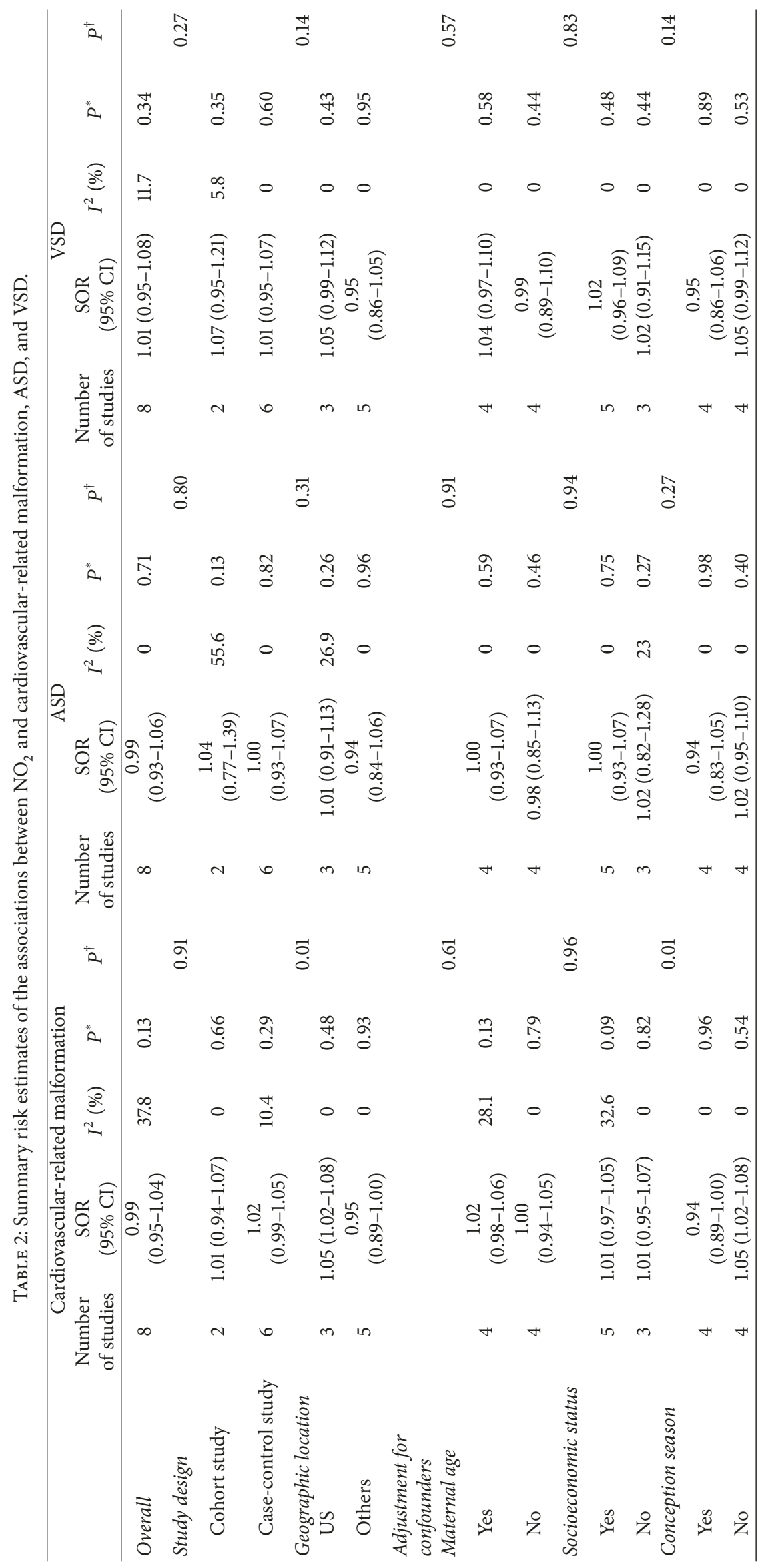




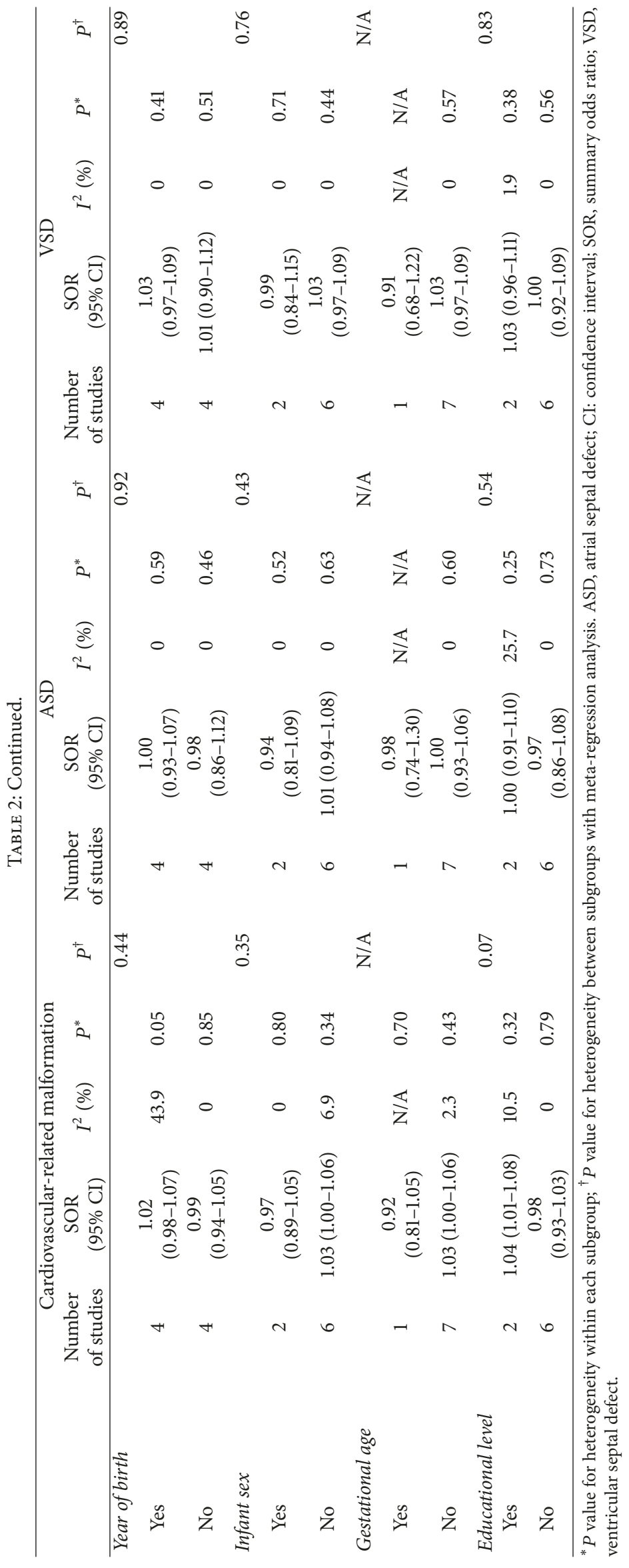




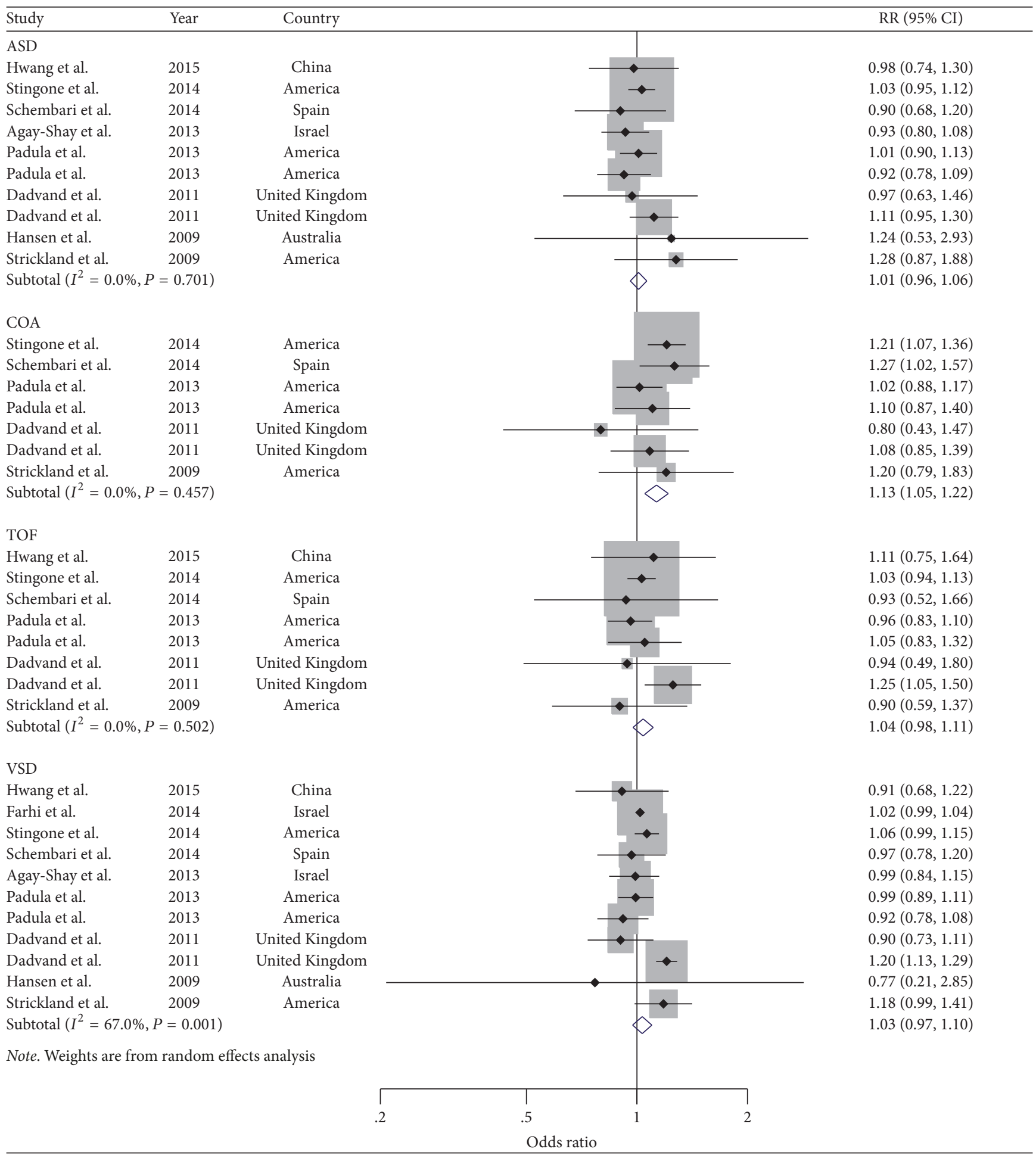

FIGURE 3: Forest plots of the relationship between $N_{x}$ exposure and risk of ASD, COA, TOF, and VSD. Squares indicate study-specific risk estimates (size of the square reflects the study-specific statistical weight); horizontal lines indicate 95\% CIs; diamond indicates the summary relative risk with its $95 \% \mathrm{CI}$.

movement [31]. Besides, another plausible explanation of information bias is residential mobility during pregnancy, which may lead to exposure misclassification. The random migration in cases and controls might generate nondifferential misclassification and decrease the accuracy of exposure assessment [27]. These reasons would more likely result in 


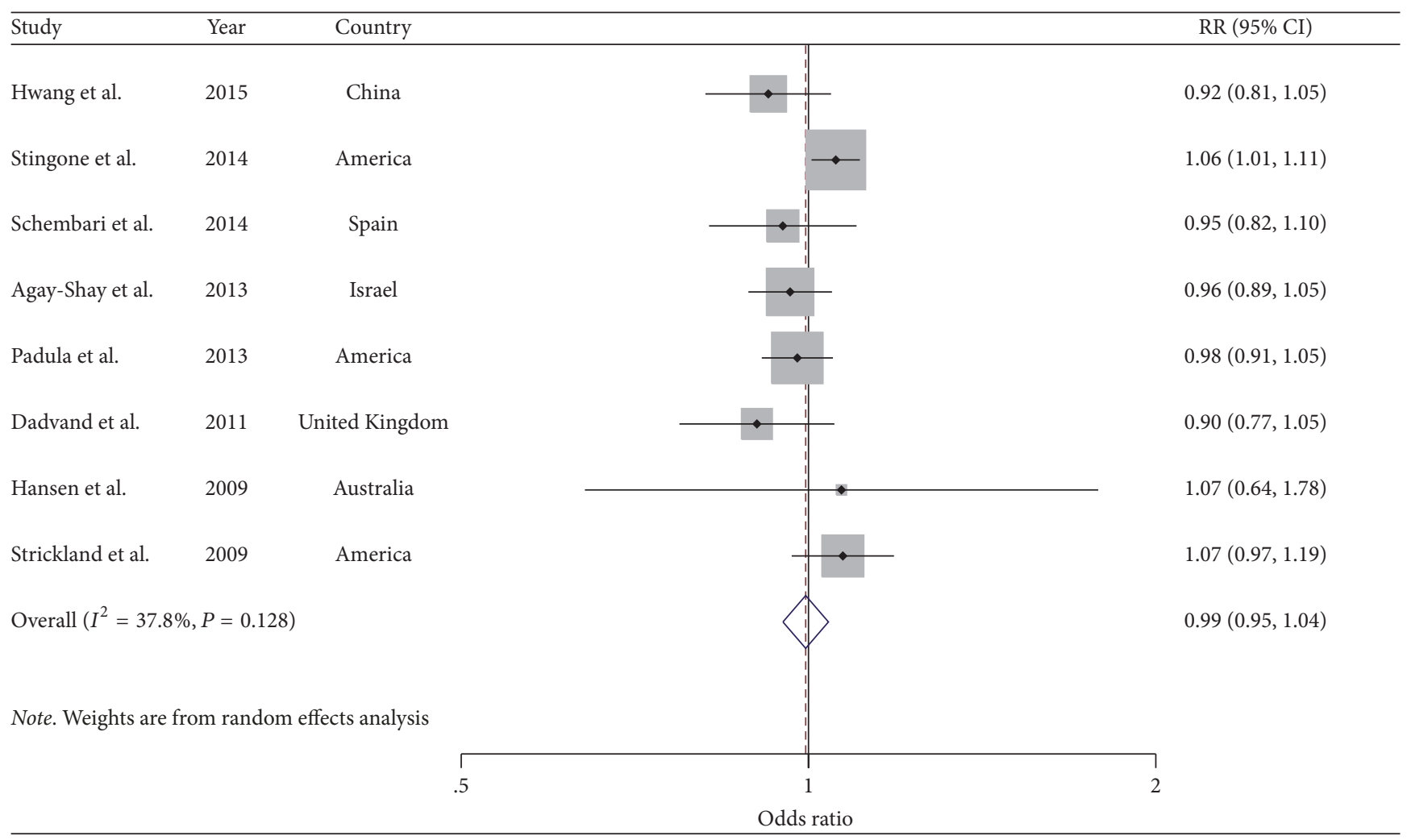

FIGURE 4: Forest plots of the relationship between $\mathrm{NO}_{2}$ exposure and risk of cardiovascular-related malformations. Squares indicate studyspecific risk estimates (size of the square reflects the study-specific statistical weight); horizontal lines indicate 95\% CIs; diamond indicates the summary relative risk with its $95 \%$ CI.

underestimation of $\mathrm{NO}_{x}$ exposure effects rather than positive results in the association.

Compared to the limited sample size of each study, our meta-analysis included a large number of cardiovascularrelated malformations cases $(n=19,079)$. This large sample size not only allowed us to investigate the association between $\mathrm{NO}_{x}$ exposure and cardiovascular-related malformations, but also facilitated for us carrying out numerous stratified and sensitivity analyses to explore the heterogeneity. Additionally, we only included the studies whose exposure period of pregnancy was first trimester or in the range of first trimester. This could help reduce the selection and detection bias of the final results.

Despite the clear strengths of this study, some limitations of our study should be acknowledged. First of all, we could not get detailed information on diagnostic tests for all cardiovascular-related malformations of included studies. Although echocardiography has been the most useful diagnostic test to confirm the presence of congenital heart defects [40], it greatly depended on the clinical skills and knowledge of operators, which might generate bias in different studies. Besides, all included studies are retrospective. The lack of follow-up could lose sight of potential cases of cardiovascular-related malformations and might lead to underestimating the total cases. Additionally, we found differences in inclusion and exclusion of cases in cardiac defects. Studies differed in their approach to classification of the same anomaly. For example, the study conducted by Strickland et al. [29] reclassified each record using a modified version of the International Pediatric and Congenital Cardiac Code implemented in the Society of Thoracic Surgeons Congenital Heart Surgery Database, while study conducted by Hwang et al. [27] classified the cardiac defect into six categories which were similar with categories used by Gilboa and colleagues [41]. Besides, other studies [7, 28, 30, 32-34] classified the cardiovascular-related malformation according to International Classification of Diseases Tenth Revision (ICD-10). This could become the largest evidence for heterogeneity for cardiac defects. Herein, we encourage further studies that should establish consistent classification standard and definition of cardiovascular-related malformation to reduce bias among studies.

Secondly, confounding factors included in the individual studies are an additional concern. In fact, there are some known or suspected risk factors for congenital anomalies including maternal age, smoking, and season of conception [42-44]. However, these potential confounders were not consistent in each study. For example, Hansen et al. [28] only noted the adjustment for neonate sex while Hwang et al. [27] and Farhi et al. [33] adjusted for seven kinds of potential confounders, respectively. Notably, some specific confounding factors such as maternal body mass index (BMI) and obesity seem to increase the risk of congenital heart defects including the septal ones [45-47]. Therefore, further studies 


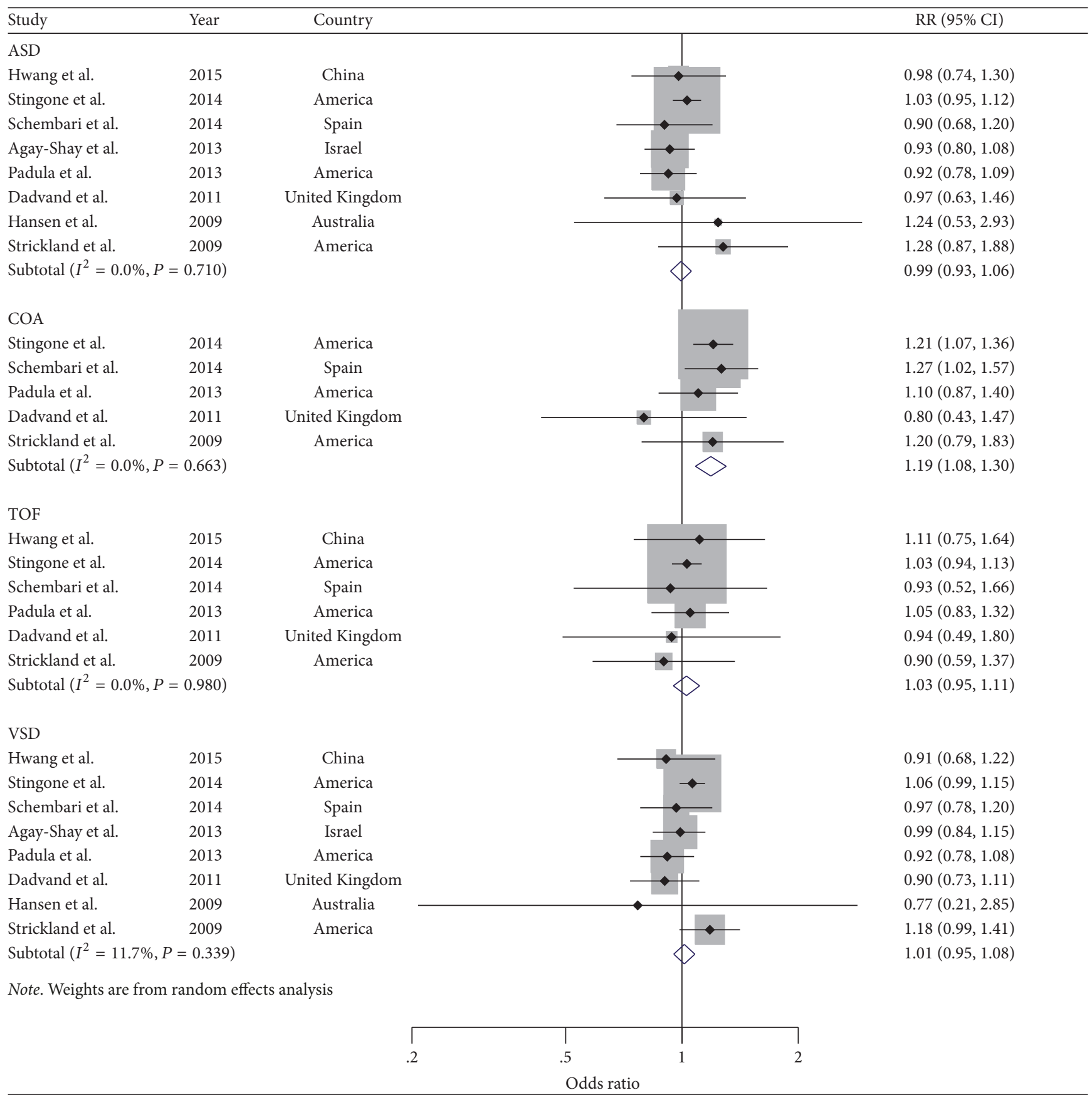

Figure 5: Forest plots of the relationship between $\mathrm{NO}_{2}$ exposure and risk of ASD, COA, TOF, and VSD. Squares indicate study-specific risk estimates (size of the square reflects the study-specific statistical weight); horizontal lines indicate 95\% CIs; diamond indicates the summary relative risk with its $95 \% \mathrm{CI}$.

should fully adjust these potential confounders or report analyses stratified by these risk factors to better be able to rule out residual confounders.

Thirdly, the results of some studies $[7,29,32]$ were based on the pregnancies reaching at least 20 weeks' gestation. Given this, these studies reported that their gestational windows of interest spanned weeks 3-7 or weeks 3-8 of pregnancy, because embryological evidence indicated the timing of specific stages of cardiac development, beginning with the migration of cells to form the endocardial tubes and culminating with the septation of the ventricles and outflow tracts in weeks 7 and 8 of development [48]. Although this period is in the range of first trimester, it will lead to underestimating harmful effect of $\mathrm{NO}_{x}$ or $\mathrm{NO}_{2}$. For example, atrioventricular septal defect, Ebstein's anomaly, and tricuspid valve dysplasia can all cause intrauterine congestive heart failure, increasing the risk of intrauterine fetal death [29]. If $\mathrm{NO}_{x}$ or $\mathrm{NO}_{2}$ could increase the risk of these malformations, in turn increasing 
the risk of abortion before week 20, we could not be able to detect these bad effects on cardiovascular-related malformations. Besides, there is experimental research showing that triggering oxidative stress in diabetic mice can result in apoptosis among migrating neural crest cells, which later results in outflow tract defects [49]. This suggests that it is possible that pollutant-induced oxidative stress in earlier weeks of development can trigger similar disruptions in neural crest cells that later affect development of cardiac structures. Further studies are warranted to explore whether windows of susceptibility to environmental insults coincide with the established stages of heart development.

Finally, in order to determine whether the exposure dose of per $10 \mathrm{ppb}$ increment is too small, we recalculated the SORs of cardiovascular-related malformation per $50 \mathrm{ppb}$ increment in $\mathrm{NO}_{x} / \mathrm{NO}_{2}$ concentration. Except for the outcome of COA and $\mathrm{NO}_{2}$ exposure, there were no significant risks of other outcomes in infants born to mothers who are exposed to $\mathrm{NO}_{x} / \mathrm{NO}_{2}$ in the first trimester. Herein, the exposure dose was not the explanation for our negative results.

\section{Conclusion}

Our meta-analysis provided the limited evidence about the relationship between $\mathrm{NO}_{x}$ or $\mathrm{NO}_{2}$ exposure in pregnant women in the first trimester and cardiovascular-related malformations, although there was a small increase risk between exposure to $\mathrm{NO}_{x} / \mathrm{NO}_{2}$ and COA malformation. Additional epidemiologic studies are warranted to provide more detailed results, including research into different kinds of cardiovascular-related malformation after better adjustment for more potential confounders.

\section{Conflicts of Interest}

The authors declare no conflicts of interest.

\section{Authors' Contributions}

Tie-Ning Zhang, Da Li, Qi-Jun Wu, and Chun-Feng Liu designed research; Tie-Ning Zhang, Da Li, Jing Xia, and QiJun Wu conducted research; Tie-Ning Zhang, Da Li, QiJun Wu, Jing Xia, and Chun-Feng Liu analyzed data; TieNing Zhang, Da Li, Qi-Jun Wu, Jing Xia, Ri Wen, Xing-Chen Chen, Ni Yang, Yan-Ling Chen, Yan-Hong Huang, and ChunFeng Liu wrote the draft; all authors read, reviewed, and approved the final manuscript. Chun-Feng Liu had primary responsibility for final content. Tie-Ning Zhang and $\mathrm{Da} \mathrm{Li}$ contributed equally to this work.

\section{Acknowledgments}

This work was supported by the Science and Technology Project of Liaoning Province (2015225025 for Yan-Hong Huang) and Science and Technology Project of Shenyang (F15-139-9-09 for Yan-Hong Huang and F13-220-9-38 for Chun-Feng Liu). This study was supported by the National Natural Science Foundation of China (no. 81372039 for
Chun-Feng Liu, no. 81402130 for Da Li, and no. 81602918 for Qi-Jun Wu), the Doctoral Start-Up Foundation of Liaoning Province (no. 201501007 for Qi-Jun Wu), and the Fok Ying Tung Education Foundation (no. 151039 for Da Li). Qi-Jun Wu was supported by the Fogarty International Clinical Research Scholars and Fellows Support Center at the Vanderbilt Institute for Global Health, funded by the Fogarty International Center, NIH, through an R24 Training Grant (D43 TW008313 to Xiao-Ou Shu).

\section{Supplementary Materials}

Supplementary Table S1: characteristics of the studies included in the meta-analysis. Supplementary Table S2: adjusted factors of the studies included in the meta-analysis. Supplementary Table S3a: methodological quality of case-control studies included in the meta-analysis. Supplementary Table S3b: methodological quality of cohort studies included in the meta-analysis. (Supplementary Materials)

\section{References}

[1] M. B. Mainolfi, H. M. Salihu, R. E. Wilson, and A. K. Mbah, "Low-level exposure to air pollution and risk of adverse birth outcomes in Hillsborough County, Florida," Journal of Occupational and Environmental Medicine, vol. 55, no. 5, pp. 490-494, 2013.

[2] M. Maisonet, A. Correa, D. Misra, and J. J. K. Jaakkola, "A review of the literature on the effects of ambient air pollution on fetal growth," Environmental Research, vol. 95, no. 1, pp. 106$115,2004$.

[3] M. Bobak, "Outdoor air pollution, low birth weight, and prematurity," Environmental Health Perspectives, vol. 108, no. 2, pp. 173-176, 2000.

[4] D. Q. Rich, K. Demissie, S.-E. Lu, L. Kamat, D. Wartenberg, and G. G. Rhoads, "Ambient air pollutant concentrations during pregnancy and the risk of fetal growth restriction," Journal of Epidemiology and Community Health, vol. 63, no. 6, pp. 488496, 2009.

[5] R. J. Šrám, B. Binková, J. Dejmek, and M. Bobak, "Ambient air pollution and pregnancy outcomes: a review of the literature," Environmental Health Perspectives, vol. 113, no. 4, pp. 375-382, 2005.

[6] M. Wilhelm and B. Ritz, "Residential proximity to traffic and adverse birth outcomes in Los Angeles County, California, 1994-1996," Environmental Health Perspectives, vol. 111, no. 2, pp. 207-216, 2003.

[7] K. Agay-Shay, M. Friger, S. Linn, A. Peled, Y. Amitai, and C. Peretz, "Air pollution and congenital heart defects," Environmental Research, vol. 124, pp. 28-34, 2013.

[8] M. E. Pierpont, C. T. Basson, D. W. Benson Jr. et al., "Genetic basis for congenital heart defects: Current knowledge - A scientific statement from the American Heart Association Congenital Cardiac Defects Committee, Council on Cardiovascular Disease in the Young," Circulation, vol. 115, no. 23, pp. 3015-3038, 2007.

[9] J. A. Connor, K. Gauvreau, and K. J. Jenkins, "Factors associated with increased resource utilization for congenital heart disease," Pediatrics, vol. 116, no. 3, pp. 689-695, 2005.

[10] K. J. Jenkins, A. Correa, J. A. Feinstein et al., "Noninherited risk factors and congenital cardiovascular defects: Current 
knowledge-a scientific statement from the American Heart Association Council on Cardiovascular Disease in the Young," Circulation, vol. 115, no. 23, pp. 2995-3014, 2007.

[11] B. Khoshnood, C. De Vigan, V. Vodovar et al., "Trends in prenatal diagnosis, pregnancy termination, and perinatal mortality of newborns with congenital heart disease in France, 1983-2000: A population-based evaluation," Pediatrics, vol. 115, no. 1, pp. 95101, 2005.

[12] K.-S. Lee, B. Khoshnood, L. Chen, S. N. Wall, W. J. Cromie, and R. L. Mittendorf, "Infant mortality from congenital malformations in the United States, 1970-1997," Obstetrics \& Gynecology, vol. 98, no. 4, pp. 620-627, 2001.

[13] D. Van der Linde, E. E. M. Konings, M. A. Slager et al., "Birth prevalence of congenital heart disease worldwide: a systematic review and meta-analysis," Journal of the American College of Cardiology, vol. 58, no. 21, pp. 2241-2247, 2011.

[14] M. D. Reller, M. J. Strickland, T. Riehle-Colarusso, W. T. Mahle, and A. Correa, "Prevalence of congenital heart defects in metropolitan Atlanta, 1998-2005," Journal of Pediatrics, vol. 153, no. 6, pp. 807-813, 2008.

[15] J. J. Nora and A. H. Nora, "The evolution of specific genetic and environmental counseling in congenital heart diseases," Circulation, vol. 57, no. 2, pp. 205-213, 1978.

[16] S. V. Glinianaia, J. Rankin, R. Bell, T. Pless-Mulloli, and D. Howel, "Particulate air pollution and fetal health: a systematic review of the epidemiologic evidence," Epidemiology, vol. 15, no. 1, pp. 36-45, 2004.

[17] M. Kampa and E. Castanas, "Human health effects of air pollution," Environmental Pollution, vol. 151, no. 2, pp. 362-367, 2008.

[18] M. Vrijheid, D. Martinez, S. Manzanares et al., "Ambient air pollution and risk of congenital anomalies: A systematic review and meta-analysis," Environmental Health Perspectives, vol. 119, no. 5, pp. 598-606, 2011.

[19] E. K.-C. Chen, D. Zmirou-Navier, C. Padilla, and S. Deguen, "Effects of air pollution on the risk of congenital anomalies: A systematic review and meta-analysis," International Journal of Environmental Research and Public Health, vol. 11, no. 8, pp. 7642-7668, 2014.

[20] D. F. Stroup, J. A. Berlin, S. C. Morton et al., "Meta-analysis of observational studies in epidemiology: a proposal for reporting," Journal of the American Medical Association, vol. 283, no. 15, pp. 2008-2012, 2000.

[21] S.-Y. Gao, Q.-J. Wu, T.-N. Zhang et al., "Fluoxetine and congenital malformations: a systematic review and meta-analysis of cohort studies," British Journal of Clinical Pharmacology, vol. 83, no. 10, pp. 2134-2147, 2017.

[22] T.-N. Zhang, S.-Y. Gao, Z.-Q. Shen et al., "Use of selective serotonin-reuptake inhibitors in the first trimester and risk of cardiovascular-related malformations: A meta-analysis of cohort studies," Scientific Reports, vol. 7, Article ID 43085, 2017.

[23] Z.-Q. Shen, S.-Y. Gao, S. X. Li et al., "Sertraline use in the first trimester and risk of congenital anomalies: a systemic review and meta-analysis of cohort studies," British Journal of Clinical Pharmacology, vol. 83, no. 4, pp. 909-922, 2017.

[24] S. Greenland and M. P. Longnecker, "Methods for trend estimation from summarized dose-response data, with applications to meta-analysis," American Journal of Epidemiology, vol. 135, no. 11, pp. 1301-1309, 1992.

[25] N. Orsini, R. Li, A. Wolk, P. Khudyakov, and D. Spiegelman, "Meta-analysis for linear and nonlinear dose-response relations: examples, an evaluation of approximations, and software,"
American Journal of Epidemiology, vol. 175, no. 1, pp. 66-73, 2012.

[26] R. DerSimonian and N. Laird, "Meta-analysis in clinical trials," Controlled Clinical Trials, vol. 7, no. 3, pp. 177-188, 1986.

[27] B.-F. Hwang, Y. L. E. Lee, and J. J. K. Jaakkola, "Air Pollution and the Risk of Cardiac Defects: A Population-Based Case-Control Study," Medicine, vol. 94, no. 44, article e1883, 2015.

[28] C. A. Hansen, A. G. Barnett, B. B. Jalaludin, and G. G. Morgan, "Ambient air pollution and birth defects in Brisbane, Australia," PLoS ONE, vol. 4, no. 4, Article ID e5408, 2009.

[29] M. J. Strickland, M. Klein, A. Correa et al., "Ambient air pollution and cardiovascular malformations in Atlanta, Georgia, 1986-2003," American Journal of Epidemiology, vol. 169, no. 8, pp. 1004-1014, 2009.

[30] A. M. Padula, I. B. Tager, S. L. Carmichael et al., "Ambient air pollution and traffic exposures and congenital heart defects in the san joaquin Valley of California," Paediatric and Perinatal Epidemiology, vol. 27, no. 4, pp. 329-339, 2013.

[31] J. A. Stingone, T. J. Luben, J. L. Daniels et al., "Maternal exposure to criteria air pollutants and congenital heart defects in offspring: Results from the National Birth Defects Prevention Study," Environmental Health Perspectives, vol. 122, no. 8, pp. 863-872, 2014.

[32] P. Dadvand, J. Rankin, S. Rushton, and T. Pless-Mulloli, “Ambient air pollution and congenital heart disease: A register-based study," Environmental Research, vol. 111, no. 3, pp. 435-441, 2011.

[33] A. Farhi, V. Boyko, J. Almagor et al., "The possible association between exposure to air pollution and the risk for congenital malformations," Environmental Research, vol. 135, pp. 173-180, 2014.

[34] A. Schembari, M. J. Nieuwenhuijsen, J. Salvador et al., "Trafficrelated air pollution and congenital anomalies in Barcelona," Environmental Health Perspectives, vol. 122, no. 3, pp. 317-323, 2014.

[35] M. Egger, G. D. Smith, M. Schneider, and C. Minder, "Bias in meta-analysis detected by a simple, graphical test," British Medical Journal, vol. 315, no. 7109, pp. 629-634, 1997.

[36] C. B. Begg and M. Mazumdar, "Operating characteristics of a rank correlation test for publication bias," Biometrics, vol. 50, no. 4, pp. 1088-1101, 1994.

[37] M. Wilhelm and B. Ritz, "Local variations in CO and particulate air pollution and adverse birth outcomes in Los Angeles County, California, USA," Environmental Health Perspectives, vol. 113, no. 9, pp. 1212-1221, 2005.

[38] A. Baccarelli and V. Bollati, "Epigenetics and environmental chemicals," Current Opinion in Pediatrics, vol. 21, no. 2, pp. 243251, 2009.

[39] L. Hou, D. Wang, and A. Baccarelli, "Environmental chemicals and microRNAs," Mutation Research - Fundamental and Molecular Mechanisms of Mutagenesis, vol. 714, no. 1-2, pp. 105-112, 2011.

[40] K. J. Mitchell and C. C. Schwarzwald, "Echocardiography for the Assessment of Congenital Heart Defects in Calves," Veterinary Clinics of North America - Food Animal Practice, vol. 32, no. 1, pp. 37-54, 2016.

[41] S. M. Gilboa, P. Mendola, A. F. Olshan et al., "Relation between ambient air quality and selected birth defects, seven county study, Texas, 1997-2000," American Journal of Epidemiology, vol. 162, no. 3, pp. 238-252, 2005.

[42] A. Hackshaw, C. Rodeck, and S. Boniface, "Maternal smoking in pregnancy and birth defects: A systematic review based on 
173687 malformed cases and 11.7 million controls," Human Reproduction Update, vol. 17, no. 5, pp. 589-604, 2011.

[43] M. Loane, H. Dolk, and J. K. Morris, "Maternal age-specific risk of non-chromosomal anomalies," BJOG: An International Journal of Obstetrics \& Gynaecology, vol. 116, no. 8, pp. 1111-1119, 2009.

[44] A. de la Vega and R. Lopez-Cepero, "Seasonal variations in the incidence of some congenital anomalies in Puerto Rico based on the timing of conception," Puerto Rico Health Sciences Journal, vol. 28, no. 2, pp. 121-125, 2009.

[45] J. Brite, S. K. Laughon, J. Troendle, and J. Mills, "Maternal overweight and obesity and risk of congenital heart defects in offspring," International Journal of Obesity, vol. 38, no. 6, pp. 878-882, 2014.

[46] J. Yang, S. L. Carmichael, M. Canfield, J. Song, and G. M. Shaw, "Socioeconomic status in relation to selected birth defects in a large multicentered US case-control study," American Journal of Epidemiology, vol. 167, no. 2, pp. 145-154, 2008.

[47] H. M. Rivera, K. J. Christiansen, and E. L. Sullivan, "The role of maternal obesity in the risk of neuropsychiatric disorders," Frontiers in Neuroscience, vol. 9, article 194, 2015.

[48] A. C. Gittenberger-De Groot, M. M. Bartelings, M. C. Deruiter, and R. E. Poelmann, "Basics of cardiac development for the understanding of congenital heart malformations," Pediatric Research, vol. 57, no. 2, pp. 169-176, 2005.

[49] S. C. Morgan, F. Relaix, L. L. Sandell, and M. R. Loeken, "Oxidative stress during diabetic pregnancy disrupts cardiac neural crest migration and causes outflow tract defects," Birth Defects Research Part A - Clinical and Molecular Teratology, vol. 82, no. 6, pp. 453-463, 2008. 


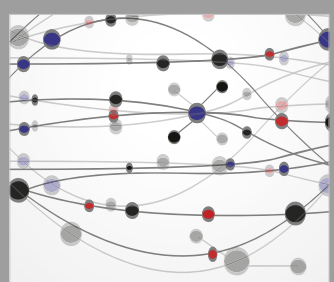

The Scientific World Journal
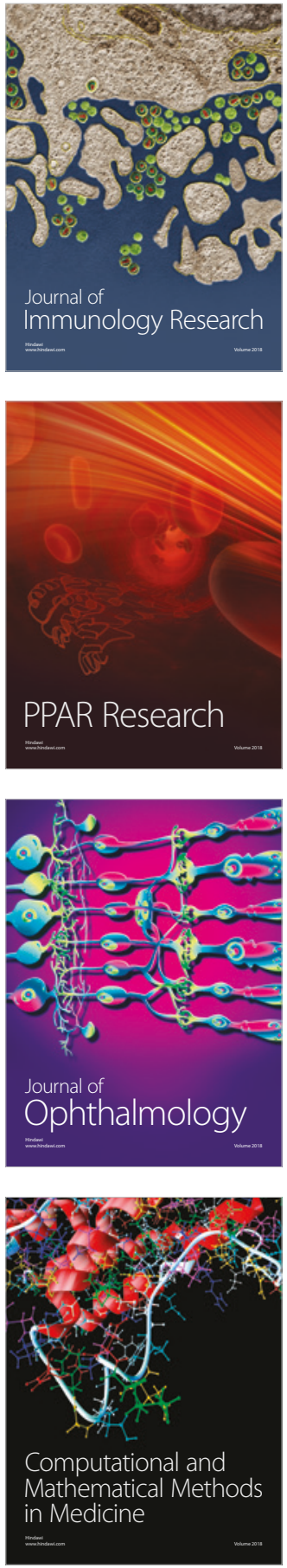

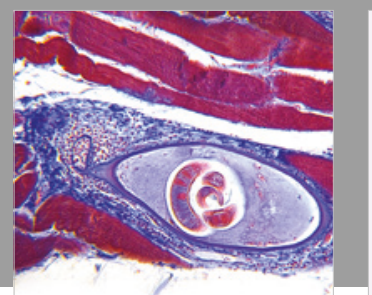

Gastroenterology Research and Practice

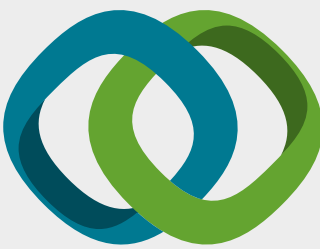

\section{Hindawi}

Submit your manuscripts at

www.hindawi.com
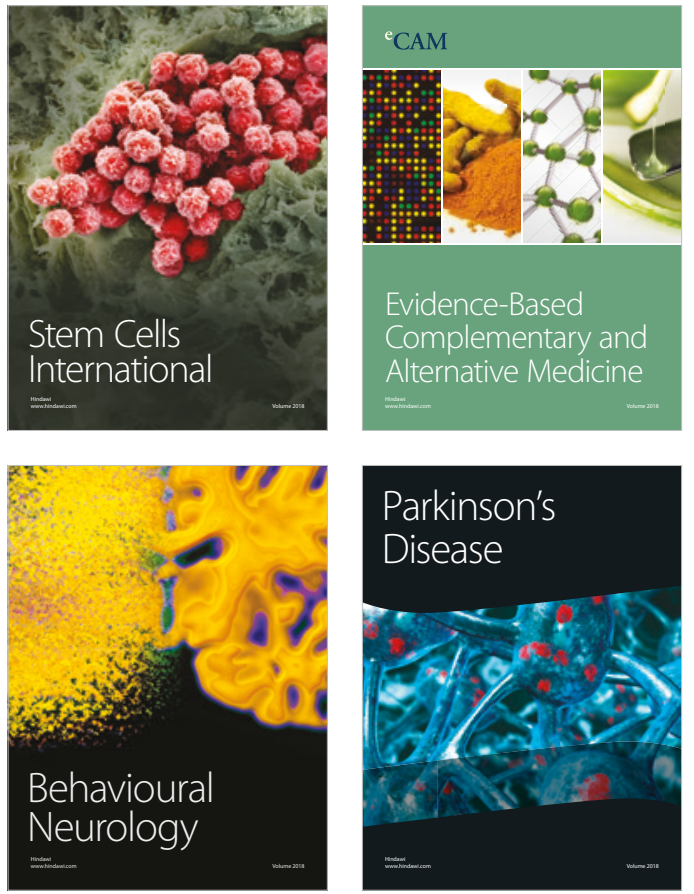

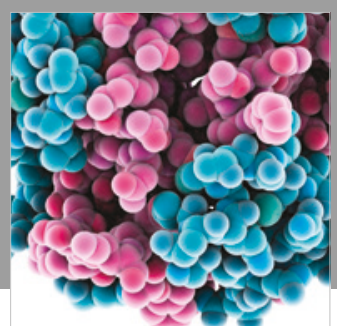

ournal of

Diabetes Research

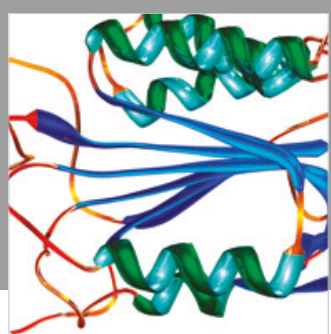

Disease Markers
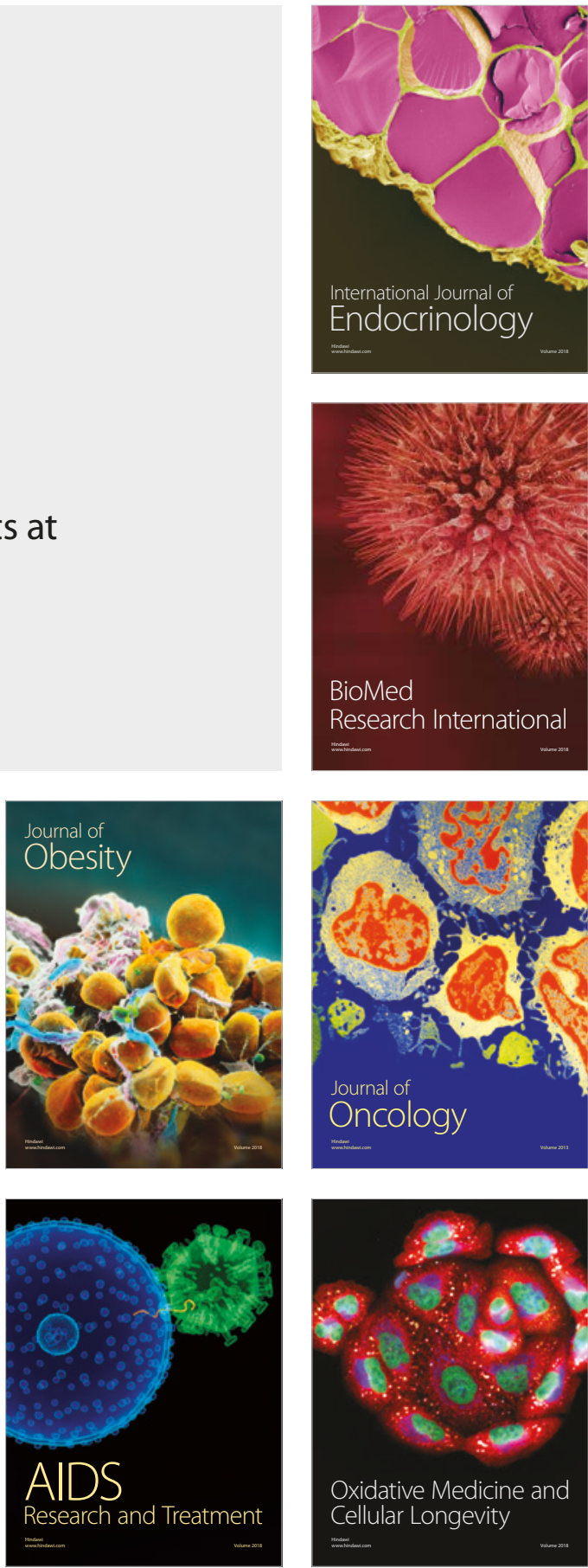